\title{
1 Ambulatory Clinic Exam Room Design with respect to Computing Devices: A Laboratory \\ 2 Simulation Study
}

\section{OCCUPATIONAL APPLICATIONS}

5 When comparing a typical exam room layout to the Department of Veterans Affairs (VA's) new exam

9 clinical workflow and spent significantly more time in screen sharing activities with the patient. A more

10 thoughtful design of the exam room layout with respect to the placement and physical design of the

11 computing set-up may reduce provider cognitive effort and enhance aspects of patient centeredness by

12 viewing the computer and electronic health record (EHR) it displays as an important mediator between

13 provider and patient. This was achieved by using an all-in-one computer attached to a wall mount that

14 moves the monitor along three axes, allowing for optimal screen positioning and adjustable depending 15 upon the scenario.

16

17 


\section{TECHNICAL ABSTRACT}

Background: Challenges persist regarding how to integrate computing effectively into the exam room,

41 while maintaining patient-centered care.

42 Purpose: Our objective was to evaluate a new exam room design with respect to the computing layout,

43 which included a wall-mounted monitor for ease of (re)-positioning.

44 Methods: In a lab-based experiment, 28 providers used prototypes of the new and older "legacy"

45 outpatient exam room layouts in a within-subject comparison using simulated patient encounters. We

46 measured efficiency, errors, workload, patient-centeredness (proportion of time the provider was focused

47 on the patient), amount of screen sharing with the patient, workflow integration, and provider situation

48 awareness.

49 Results: There were no statistically significant differences between the exam room layouts for efficiency,

50 errors, or time spent focused on the patient. However, when using the new layout providers spent $75 \%$

51 more time in screen sharing activities with the patient, had $31 \%$ lower workload, and gave higher ratings

52 for situation awareness (14\%) and workflow integration (17\%).

53 Conclusions: Providers seemed to be unwilling to compromise their focus on the patient when the

54 computer was in a fixed position in the corner of the room and, as a result, experienced greater workload,

55 lower situation awareness, and poorer workflow integration when using the old "legacy" layout. A

56 thoughtful design of the exam room with respect to the computing may positively impact providers'

57 workload, situation awareness, time spent in screen sharing activities, and workflow integration.

59 KEYWORDS: Human-computer interaction, Computer workstations, Mental workload, Exam room

60 design; Exam room computing; Patient centeredness 


\section{INTRODUCTION}

Substantial research has evaluated the impact of the electronic health record (EHR) on the

63 provider-patient interaction in ambulatory care. However, challenges persist regarding how to best

64 integrate the electronic health record (EHR) into patient visits and clinical workflow, without

65 adversely influencing the provider-patient interaction and relationship (Patel, Vichich, Lang, Lin, \&

66 Zheng, 2017; Saleem et al., 2014). With respect to integrating computerized applications into the

67 patient visit while maintaining patient-centeredness, the computer and EHR should be viewed as a

68 "third party" and should serve as a mediator between provider and patient (Saleem et al., 2014). This

69 viewpoint counters an existing viewpoint that suggests the integration of computers/EHR negatively

70 impacts patient-centeredness due to the exam room layout and the inability of this integration to

71 effectively substitute for current paper-based clinical workflows (Saleem et al., 2014). Integrating

72 EHRs into the patient visit, while maintaining patient-centeredness, may thereby help enhance, rather

73 than negatively impact, the provider-patient relationship.

Various practices are responsible for optimal integration of computers into exam rooms. A

75 systematic review of prior research found that multiple studies support practices that utilize the

76 computer through sharing the computer and what is on the screen, adjusting room design, and verbal

77 and nonverbal communication (Patel et al., 2017). However, when the EHR is introduced and used in

78 provider-patient encounters, the provider-patient relationship is affected by both the provider's body

79 orientation (Frankel, 2016; Pearce, Dwan, Arnold, Phillips, \& Trumble, 2009) and the patient's

80 behaviors with the computer (Pearce, Arnold, Phillips, Trumble, \& Dwan, 2011). In one study, the

81 provider's body orientation was classified as either 'unipolar' or 'bipolar'; where 'unipolar'

82 orientation classified the provider's body as oriented towards the computer, and 'bipolar'

83 classification indicated the provider's body orientation fluctuated between facing the patient and the

84 computer (Pearce et al., 2009). The behavior of the patient with the computer and EHR in the room

85 was classified as having three components: 'screen watching', 'screen ignoring', and 'screen

86 excluding' to try and influence the provider's actions (Pearce et al., 2011). A recent study 
87 demonstrated that patients looked at the computer twice as much when the screen was within their 88 gaze, and that the EHR was used for a consistent proportion of the interaction (Kumarapeli \& de, 89 2013). Therefore, if increased provider-patient interaction is desired with the inclusion of the EHR or 90 computer, there is a need for specific layout guidelines to induce interaction and facilitate the 91 computer's role in the interaction.

92 Computers are often placed wherever proper wiring is available and often this positioning 93 affected communication (Ventres et al., 2006). Previous studies have focused on how computer use 94 affects interactions between providers and patients in exam room settings (McGrath, Arar, \& Pugh, 95 2007; Patel et al., 2017; Rouf, Whittle, Lu, \& Schwartz, 2007). Through a systematic review, it 96 appears that a gap in research exists when evaluating the practice of room design through randomized 97 controlled trials, and most studies reviewed were of the observational variety (Patel et al., 2017). 98 McGraph et al. (2007) found three different office spatial designs: 'open,' 'closed' and 'blocked'. An 99 'open' orientation has the physician oriented toward the patient, even when using the computer and 100 the 'closed' orientation was described as the physician with their back turned to the patient while 101 using the computer. Finally, the 'blocked' orientation was described as the physician oriented toward 102 the patient, but the computer monitor obstructing the view between the physician and patient. The 103 'open' arrangement put physicians in a position to establish better eye contact and physical orientation 104 than did the other configurations.

105 This study was completed to obtain empirical evidence regarding provider preference and 106 performance differences when using a more tangible and interchangeable exam room layout. An 107 additional aim was to support the notion that a redesigned exam room layout has various benefits for 108 the provider-patient relationship. To do this, we designed and conducted a study comparing two 109 layouts (current version 'A' vs new version 'B'). The former had a desktop computer, placed in the 110 corner of the room (Figure 1), while the latter included an all-in-one computer attached to a wall111 mounted armature system that was adjustable along three axes (Figure 2), making it easier for 112 providers to achieve an 'open' position (McGrath et al., 2007). Layout A, with the computer monitor 
113 placed on a desk in a corner of the room, is a typical arrangement in practice, especially when

114 computers were initially introduced into exam rooms (Frankel et al., 2005; Frankel \& Saleem, 2013).

115 The impact of the placement of exam room computers on provider-patient communication, both verbal

116 and non-verbal, was not considered in many cases (McGraph et al., 2007), resulting in a convenience-

117 based placement of the computer (e.g., by the nearest electrical outlet). Based upon the flexibility and

118 maneuverability offered by the set-up in the new layout, we expected layout B to result in greater

119 efficiency and accuracy, increased evidence of patient centeredness, better alignment with the providers'

120 clinical workflow, enhanced perceived situation awareness, and a decrease in perceived workload.

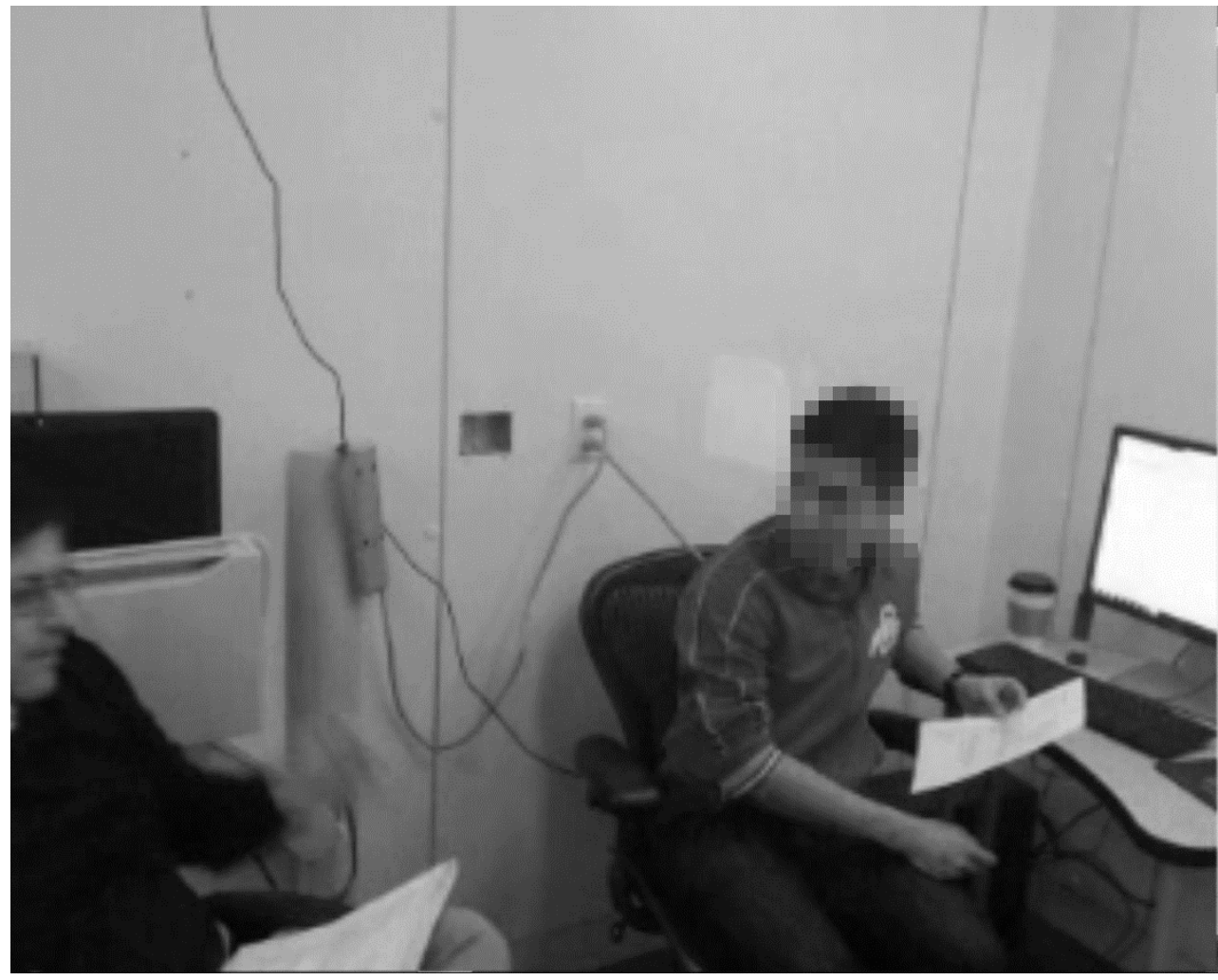

124 Figure 1: Current design, layout A, with the computer workstation on a fixed desk in the corner of the room. 


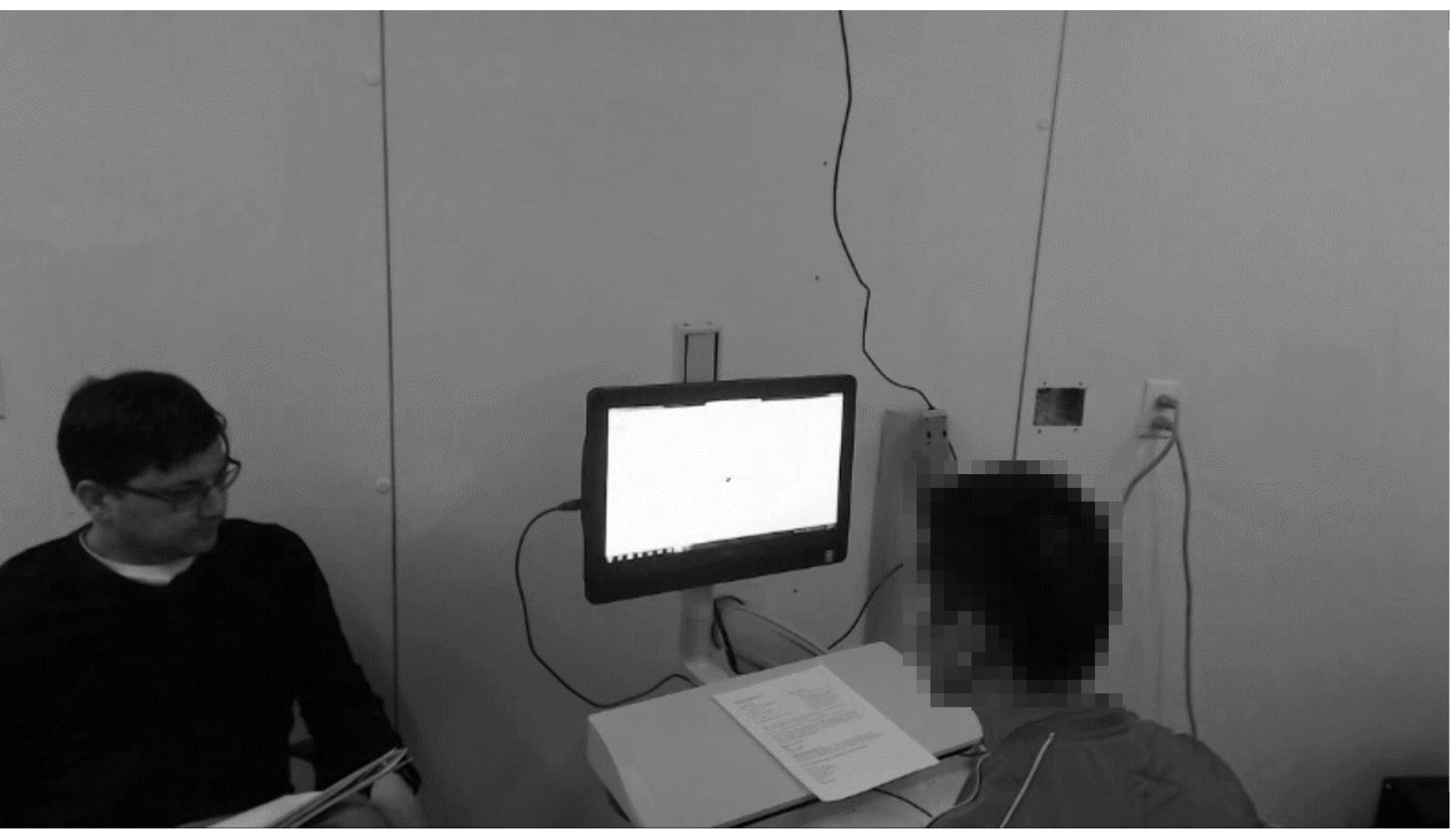

127 Figure 2: New design, layout B, with a wall-mounted armature system for the computer monitor.

\section{METHODS}

\subsection{Participants}

An a priori power analysis was completed, based on the primary outcome measure of workload,

133 as measured by the NASA-Task Load Index (TLX) (Hart \& Staveland, 1988). From our previous studies

134 that measured human performance, we estimated the standard deviation of NASA TLX workload scores

135 as 13.2. The NASA TLX has a range of 100 points, and a difference of 10 points was considered a

136 relevant difference. Assuming respective Type I and Type II error rates as 0.05 and 0.20 , the required

137 sample size is 28 participants to have $80 \%$ power for detecting a 10-point difference between the current 138 design and the redesign.

139 A total of 28 healthcare providers (17 male, 11 female) completed the study, with the mean age 140 being 31 (range: 26-59). Using a convenience sampling method, four attending physicians, 23 resident 141 physicians, and one nurse practitioner were recruited. In total, 26 of the 28 providers used the

142 Department of Veterans Affairs (VA) Computerized Patient Record System (CPRS) as their EHR often or 
143 occasionally; the majority of the providers were resident physicians who had previously rotated through

144 the VA and had used CPRS. Eight providers currently utilize a wall-mounted armature system in the

145 exam room, five providers currently utilize a stationary desktop, six utilize a laptop, seven do not utilize a

146 computer in any capacity, one utilizes a computer on wheels, and one provider did not provide a response.

147 All providers had experience working with patients in an outpatient examination room, with 24 providers

148 being employed through the University of Louisville, two through an independent family practice, one

149 through the Baptist Health Center, and one from the Louisville VA Medical Center.

\subsection{New exam room design}

Our redesigned exam room layout with respect to the computing is based on the VA's new exam

152 room design standard. The redesigned exam room includes a mobile computing work station with an

153 armature system and a moveable table that can rotate against the wall or rotate out to form a consult

154 surface for a keyboard or printed materials that can be viewed with the patient. Historically, computers

155 were introduced into the exam rooms with the desk and computer fixed to the wall in a way that

156 potentially encouraged the clinician to turn their back to the patient while using the EHR. The VA Office

157 of Construction \& Facilities Management decided that the new exam room design should minimize the

158 dependency of a built-in desk, which seemed to encourage a 'move-in and occupy' mindset. The new

159 exam room was designed with built-in efficiency, encouraging the provider to move from one exam room

160 to another, which is consistent with the new team-based models of care (Helfrich et al., 2016), where

161 members of the healthcare team rotate to the patient in a single location. We simulated this new exam

162 room design in our laboratory, as well and the older exam room design with a computer on a desk against

163 a wall.

\section{2.3. Experimental design}

165 We used a single-factor, within-subjects experimental design. The single factor was 'Type of

166 Exam Room Layout' with two levels (A, B), one representing a current, typical exam room layout (A),

167 and the other representing the redesigned layout, where the EHR/computer is designed to be more easily

168 incorporated with the provider-patient interaction (B). The presentation order of designs A and B were 
counterbalanced to account for potential crossover effects. Dependent measures addressed efficiency,

170 errors, workload, patient-centeredness, screen sharing, workflow integration, and situation awareness.

171 Table 1 lists and defines the outcome measures, and describes what data collection tool or method was

172 used for each.

173 Table 1 - Outcome measures for comparing a current, typical exam room layout with the redesigned

174 layout during lab simulation study.

\begin{tabular}{|c|c|c|}
\hline Outcome measure & Definition & Measuring tool / method \\
\hline Efficiency & $\begin{array}{l}\text { Efficiency completing scenarios with the } \\
\text { given exam room and computing layout. }\end{array}$ & Time to complete test scenarios \\
\hline Errors & $\begin{array}{l}\text { Deviations or omissions from the given } \\
\text { clinical scenarios. }\end{array}$ & $\begin{array}{l}\text { Completeness of each clinical } \\
\text { scenario. }\end{array}$ \\
\hline Workload & $\begin{array}{l}\text { The difference between the amount of } \\
\text { resources available within a person and the } \\
\text { amount of resources demanded by the task } \\
\text { situation (Sanders \& McCormick, 1993) }\end{array}$ & $\begin{array}{l}\text { NASA Task Load Index (TLX) } \\
\text { (Hart \& Staveland, 1988) }\end{array}$ \\
\hline $\begin{array}{l}\text { Patient- } \\
\text { centeredness }\end{array}$ & $\begin{array}{l}\text { Time the provider is focused on the patient } \\
\text { compared to the computer }\end{array}$ & $\begin{array}{l}\text { Eye gaze (E.; Montague \& Asan, } \\
\text { 2014; E.; Montague et al., 2011) }\end{array}$ \\
\hline $\begin{array}{l}\text { Amount of screen } \\
\text { sharing with the } \\
\text { patient }\end{array}$ & $\begin{array}{l}\text { Time spent sharing information from the } \\
\text { EHR and related software programs where } \\
\text { both the provider and patient are viewing the } \\
\text { computer monitor }\end{array}$ & $\begin{array}{l}\text { Time spent during screen sharing } \\
\text { activities. }\end{array}$ \\
\hline $\begin{array}{l}\text { Workflow } \\
\text { integration of } \\
\text { computer/EHR }\end{array}$ & $\begin{array}{l}\text { Degree to which new technology is tailored } \\
\text { such that it fits into the clinician's workflow } \\
\text { process for delivering patient care }\end{array}$ & $\begin{array}{l}\text { Workflow Integration Survey (WIS) } \\
\text { (Flanagan et al., 2011) }\end{array}$ \\
\hline
\end{tabular}




\begin{tabular}{|c|c|c|}
\hline $\begin{array}{l}\text { Situation } \\
\text { Awareness }\end{array}$ & $\begin{array}{l}\text { Perception and comprehension of elements } \\
\text { in the environment; projection of their status } \\
\text { in the future (Endsley, 1995) }\end{array}$ & $\begin{array}{l}\text { Situation Awareness Rating } \\
\text { Technique (SART) (Selcon \& } \\
\text { Taylor, 1990) }\end{array}$ \\
\hline
\end{tabular}

175

For efficiency, errors, patient-centeredness, and screen sharing, data were collected by using video recordings and screen captures from Morae software (version 3.3.4, TechSmith Corporation, Okemos, MI). Specifically, time to complete a scenario (efficiency) was measured through a task-timing function with video recordings, while errors were measured by evaluating screen captures of the provider's CPRS inputs and video recording from two cameras. One camera facing the provider and patient, and the other attached atop the exam room computing device, respectively captured screen sharing and patient-centeredness. Data for the NASA-TLX was collected via a computer-based survey with a scale of 1-100. The WIS and SART were paper-based measurements based on a scale of 1-5 and 17 , respectively.

\subsection{Procedure}

Providers were brought to the Center for Ergonomics laboratory and they read an IRB-approved informed consent form. A brief overview of the study was described to the provider followed by a fiveminute guided familiarization session with the EHR used for the study, the VA's CPRS. Upon completion of the familiarization session, the first testing session began by working through one of two potential scenarios and layouts. Care was taken by the facilitator not to refer to the exam room layouts as "old" and "new", which instead were referred to as "first" and "second". Once the session was completed, or the 20-minute time limit was met, the provider left the simulation area to complete the paper-based SART and WIS, as well as the computer-based NASA-TLX. The provider was brought back into the simulation area to complete the second session using the alternative layout (i.e., the provider's second simulated scenario and layout was different that the first). Similar to the first event, once the scenario was completed, or the 20 -minute time limit was met, the provider left the simulation area to complete the SART, WIS, and NASA-TLX. Finally, the provider was guided through a semi-structured debrief session 
to gather any final thoughts pertaining to the study. See Appendix 1 for the semi-structured interview

200 guide. After the debrief session was concluded, the provider was compensated and dismissed. The entire

201 session was designed not exceed 90 minutes in total.

202

203

204

205

206

207

208

209

210

211

212

213

214

215

216

217

218

219

220

221

222

223

224 the scenarios.

\subsection{Simulation scenarios}

We used similar outpatient visit scenarios for the provider to complete using both room layouts (A and B). These scenarios were reviewed and revised by a physician consultant to ensure a sufficient level of realism. Fictitious patient records for our scenarios were entered into the demo version of CPRS and populated with the scenario data, including historical and current vitals, a previous progress note, and medication list. A member of the study team [JJS] played the part of the patient. The patient actor asked for similar actions from the provider regardless of the layout and scenario. That is, regardless of the scenario or layout, the patient actor gave the provider a list of current medications and asked to see a history of vital readings from previous visits (blood pressure or respiratory rate depending on the specific patient scenario) to show interest in looking at their EHR record. The scenarios only differed in 'surfacelevel' aspects such as fictitious patient name, similar chief complaint, similar co-morbidities, similar medications, etc. However, the scenarios required providers to complete the same tasks, including creating a progress note, sharing lab results with the patient, medication reconciliation, ordering / renewing medications, and other common tasks associated with a primary care visit. Providers were asked to complete the clinical tasks; no instructions were given to the providers regarding patient-centeredness and screen sharing. The presentation order of the two patient scenarios was counterbalanced across layouts A and B (in addition to the layouts being counterbalanced across providers). In other words, the first provider used layout A with scenario 1, then layout B with scenario 2. The second provider used layout B with scenario 1, then layout A with scenario 2. The third provider used layout A with scenario 2, then layout B with scenario 1. The fourth provider used layout B with scenario 2, then layout A with scenario 1. This counterbalancing scheme was repeated for the next 24 providers. See Appendix 2 for 


\section{2.6. Layouts A and B}

A picture of Layout A, with respect to the computing, can be seen in Figure 1, and a separate

229 picture of Layout B, with respect to the computing, can be seen in Figure 2. Layout A has a simple

230 computer and 19-inch monitor setup on a desk at the nearest electric outlet with no respect to the locale of

231 the patient, patient table, or other needed medical tools. Layout B has an all-in-one computer (19.5-inch

232 monitor) attached to a wall mount that moves the screen along three axes allowing for optimal screen

233 positioning that can be adjusted depending upon the scenario. Placement of the wall mount was

234 determined based upon where the most open space was located in the exam room to not limit the potential

235 movement of the screen along any axis. This is consistent with the VA's new exam room design standard,

236 which is the basis for Layout B. Both simulated exam rooms were of high fidelity with regard to the exam

237 room computing device, room layout, and furniture pieces. However, we did not include many smaller

238 items that are typically in exam rooms, such as a blood pressure monitor, opthalmoscope, supply cart, etc.

239

240

241

242

243

244

245

\subsection{Analysis}

Analysis was done with an A vs. B comparison of the current, typical exam room layout and the redesigned layout with statistical analyses performed to compare the measures in Table 1 across the two types of exam room layouts. Each provider completed the NASA-TLX, WIS, and SART instruments twice, once for each of the two layouts. The SART instrument for situation awareness contained 10 items that were rated on a Likert-type scale from 1-7. Each of the 10 items map to three subscales for 'understanding', 'demand', and 'supply'. A composite SART score for situation awareness (SA) was calculated using: $\mathrm{SA}=\mathrm{U}-(\mathrm{D}-\mathrm{S})$, where: $\mathrm{U}=$ summed understanding; $\mathrm{D}=$ summed demand; $\mathrm{S}=$ summed supply. Paired $t$ tests were used to compare outcomes between the two layouts when parametric assumptions were met, and Wilcoxon Signed Rank tests were used otherwise. Statistically significant differences between layouts were concluded using a significance level of 0.05 .

Debriefing responses were recorded for all 28 providers. The debrief interviews were first transcribed from audio recordings. Then, responses from the debrief interview transcripts were reviewed 
253 by a member of the study team for recurrent themes across providers. A second study team member

254 reviewed and verified the summary of interview responses for repeating patterns within the full study

255 sample. Recurrent themes centered around layout preference, provider-patient interaction, and redesign

256 recommendations.

257 The remote database supporting the demo version of CPRS was inaccessible during the last

258 provider's session. Therefore, quantitative data for this provider was not included (i.e., the sample size

259 was 27 for the statistical analyses).

\section{RESULTS}

A summary of statistical results is provided in Table 2. There were no significant differences

264 between layouts for measures of efficiency, errors, or patient centeredness. However, there were

265 significant differences for time spent in screen sharing activities, as well as provider perceived situation

266 awareness and workload between layout types.

Table 2: Results for Efficiency, Errors, Patient Centeredness, Screen Sharing, and Situation Awareness $269 \quad(n=27)$

\begin{tabular}{|l|l|l|l|l|}
\hline Outcome & Layout A - Mean & Layout B - Mean & Statistical Test & $p$-value \\
Measure & (SD) & (SD) & Used & \\
\hline Efficiency - Time & $604(202.9)$ & $585(205.0)$ & Wilcoxon Signed & 0.501 \\
scenario (seconds) & & Ranks Test & \\
\hline Errors - Number & $1(0.9)$ & $1(0.9)$ & Wilcoxon Signed & 0.529 \\
of Errors & & & Ranks Test & \\
Committed & & $128(84.5)$ & Wilcoxon Signed & 0.648 \\
\hline Patient & $139(87.7)$ & & Ranks Test & \\
\hline Centeredness & & & & \\
\hline
\end{tabular}




\begin{tabular}{|l|l|l|l|l|}
\hline (amount of time & & & & \\
focused on patient & & & & \\
\hline Patient & $22(9.2)$ & Paired T-test & 0.482 \\
Centeredness & Percentage of & & & \\
time focused on & & & & \\
patient) & & & & \\
\hline Screen Sharing & $24(20.5)$ & $25(5.7)$ & Wilcoxon Signed & $0.022^{*}$ \\
(Amount of time & & & & \\
screen sharing & & & & \\
with patient in & & & & \\
seconds) & & & & \\
\hline Situation & & & & \\
\hline Awareness & & & & \\
\end{tabular}

Note: $*$ denotes statistical significance.

For workload, five out of six of the NASA-TLX subscales significantly differed between layouts

274 (Table 3), though results for the mental workload subscale only approached significance.

Table 3: NASA-TLX Subscale Comparison of Layout A vs. B (comparisons using paired $t$ tests; $\mathrm{n}=27$ )

\begin{tabular}{|l|l|l|l|}
\hline NASA-TLX Subscale & Layout A - Mean (SD) & Layout B - Mean (SD) & $\boldsymbol{p}$-value \\
\hline Mental Workload & $53(28.7)$ & $44(25.9)$ & 0.054 \\
\hline Physical Workload & $35(28.9)$ & $16(12.0)$ & $0.003^{*}$ \\
\hline Temporal & $53(22.3)$ & $40(24.9)$ & $0.030^{*}$ \\
\hline
\end{tabular}




\begin{tabular}{|l|l|l|l|}
\hline Performance & $54(25.1)$ & $44(28.7)$ & $0.049^{*}$ \\
\hline Effort & $55(24.6)$ & $38(21.7)$ & $<0.001^{*}$ \\
\hline Frustration & $60(29.8)$ & $35(25.4)$ & $<0.001^{*}$ \\
\hline Overall Workload & $52(20.0)$ & $36(17.0)$ & $<0.001^{*}$ \\
\hline
\end{tabular}

Note: * denotes statistical significance.

Finally, three out of four subscales in the WIS were found to be significantly different between layouts (Table 4), as well as the total WIS scores, while differences in the paper workaround subscale approached significance.

Table 4: Workflow Integration Survey (WIS) analysis Layout A vs. B ( $\mathrm{n}=27)$

\begin{tabular}{|l|l|l|l|}
\hline WIS Subscale & Layout A - Mean (SD) & Layout B - Mean (SD) & $\boldsymbol{p}$-value \\
\hline Navigation & $3.5(1.0)$ & $4.0(0.8)$ & $0.008^{*}$ \\
\hline Usability & $2.6(1.2)$ & $3.4(1.0)$ & $<0.001^{*}$ \\
\hline Paper Workarounds & $3.3(1.1)$ & $3.5(1.1)$ & 0.057 \\
\hline Workload & $2.6(0.7)$ & $3.1(0.9)$ & $0.002^{*}$ \\
\hline Total & $3.0(0.8)$ & $3.5(0.8)$ & $<0.001^{*}$ \\
\hline
\end{tabular}

Note: * denotes statistical significance.

Table 5 provides a summary of the themes revealed from analysis of the semi-structured debrief interviews. Two members of the study team agreed that the debrief interviews revealed interesting concepts related to three main themes: (1) layout preference; (2) provider-patient interaction; and (3) redesign recommendations. All providers indicated a preference for layout B due to the mobility associated with the wall-mounted armature system, and because the patient was within the provider's field of view. Similarly, providers indicated that layout B facilitated provider-patient interaction because the patient was in close proximity and the provider did not experience ergonomic discomfort to interact with the patient (i.e., providers turned and contorted their torso, neck, etc. to face the patient with layout 
294 A). Finally, providers described a couple of redesign recommendations for both layouts A and B. For

295 layout A, they suggested moving the patient to a location within their field of view (i.e. next to the desk).

296 For layout B, providers recommended the wall mounted armature system be fully adjustable in a vertical

297 direction so they could stand if needed.

298 Table 5: Debrief Interview Responses; Themes and Subthemes ( $\mathrm{n}=28)$

\begin{tabular}{|l|l|}
\hline Theme & Subthemes \\
\hline Layout preference & Mobility \\
& Field of view \\
\hline Provider-patient interaction & Spatial relationship to patient \\
& Ergonomic discomfort \\
\hline Redesign recommendations & Patient location \\
& Adjustable work area \\
\hline
\end{tabular}

\section{DISCUSSION}

The academic literature supports several practices for promoting provider-patient interaction with

304 the use of exam room computing (Patel, Vichich, Lang, Lin, \& Zheng, 2017). Recommended behavioral

305 and communication practices, as supported by evidence, are: (1) using the computer to facilitate

306 conversation; (2) adjusting room design; (3) maintaining eye contact with the patient while typing; (4)

307 separating typing and patient interaction; (5) talking to the patient while gazing at the computer; (6) using

308 a postural style that allows the clinician to face the patient most of the time; (7) inviting the patient to look

309 at the screen before the patient asks; and (8) informing the patient about the functions and role of the

310 computer. Adjusting the exam room design was the focus of our study, as it is both strongly supported by

311 available research evidence and also related to other evidence-based strategies for promoting provider-

312 patient interaction. 
314 simultaneously view the record, and using computers that allow for easy repositioning of the screen

315 (Baker, Reifsteck, \& Mann, 2003; Ventres et al., 2006). Adjustable and moveable furniture have also

316 been reported to facilitate orienting the room layout to be more patient-centered (Patel et al., 2017). The

317 new exam room design used here incorporated these recommended design practices, and our findings

318 support the notion of 'using the computer to facilitate conversation', an evidence-based strategy for

319 promoting provider-patient interaction with the use of exam room computing (Patel et al., 2017). The

320 new exam room design seems to facilitate this strategy. The new design, with the ability to easily

321 reposition the monitor and easily move the workspace furniture, may also facilitate other evidence-based

322 practices for promoting provider-patient interaction such as: maintaining eye contact with the patient

323 while typing; using a postural style that allows the provider to face the patient most of the time; and

324 inviting the patient to look at the screen before the patient asks (Patel et al., 2017).

\subsection{Efficiency, Errors, and Patient Centeredness}

Objective measurements of efficiency, errors, and patient centeredness (percentage of time

330 unique with respect to related studies. Others have found that the spatial organization of the exam room,

331 including placement of the computer, could inhibit or facilitate communication (Frankel et al., 2005). The

332 arrangement tested by these authors that facilitated communication was similar to the one we used for

333 layout B, with a wall-mounted armature system for the computer monitor for ease of (re)-positioning.

334 However, while the Frankel et al. (2005) study revealed that this type of arrangement facilitated provider-

335 patient communication, their study was qualitative in nature and did not measure the efficiency of the

336 visit, errors, or time focused on the patient. Therefore, it is unclear if the providers in their study were

337 predominately focused on the patient or computer screen while communicating with the patient. One

338 study that did measure time focused on the patient compared only the use of paper-based records with an

339 EHR (Asan, Smith, \& Montague, 2014). These authors found that providers spent a significantly smaller 
340 proportion of time gazing at the patient when using an EHR compared with when using a paper chart.

341 One interpretation for the lack of a substantial difference in our study is that neither layout helps (or

342 hinders) a provider's performance in these measures. However, the lack of a clear difference may have

343 occurred due to the fact the provider did not have to rely more or less on the EHR based on the scenario.

344 Moreover, the provider could have gathered much of the needed information by interacting with the

345 patient and not with the EHR, meaning the EHR was used as more of an assistive tool to try and facilitate

346 conversations between the provider and patient. Since the EHR was not used as a crutch for the provider's

347 performance, the provider could dictate how much EHR use would be incorporated in the patient visit.

348 The amount of such use is variable, and thus may have led to the lack of significant differences in time,

349 number of errors, and amount of time focused specifically on the patient.

350

351

352

353

354

355

356

357

358

359

360

361

362

363

364

365

366

\subsection{Workload}

We believe the current study is the first to measure changes in perceived workload with different exam room layouts. Layout B was more favorable in terms of perceived physical workload, temporal workload, performance, effort, and frustration. Despite the performance results of the NASA-TLX favoring layout B, performance measures (time and errors) showed no significant differences. However, some of the comments given during the debriefing match these findings. Providers complained about the amount of physical movement and general discomfort encountered while using layout A. The most common complaints were about having to turn around constantly to shift attention between the EHR and patient, twisting at the waist to look over their shoulder to check on patient while interacting with the EHR, and having their back turned towards the patient. Constantly adjusting the body posture to accommodate the EHR and patient is a logical explanation for the less favorable physical workload ratings for layout A. Additionally, providers mentioned they felt rude by having their back turned to the patient and layout A would have been easier if they took paper notes. This supports the NASA-TLX scores in regards to the high frustration scores for layout A. The temporal workload, effort, and frustration subscales were significantly lower with layout B, likely because of the personalization of the layout B, which accounts for various patient locations to assist with EHR and patient attention shifting. 


\subsection{Screen Sharing}

To our knowledge, this is the first study to measure difference in the time spent in screen sharing activities between exam room layouts. Layout B led to a larger amount of time screen sharing compared to layout A. Similar to the NASA-TLX subscales, the cause of the increased amount of screen sharing in layout B is likely to be the wall-mounted system. With layout B, the computer is fully adjustable, potentially making the providers more willing to share the screen with the patient. With layout A, the only way to effectively share the screen with the patient was by relocating the patient and moving him/her to the screen, whereas with layout B the screen can be adjusted and moved to the patient by the provider. This not only promotes the increased amount of screen sharing, but also likely promotes patient centeredness. However, during the debriefing, providers expressed concern about the potential of a patient seeing information the provider did not intend to share. This concern is consistent with another study (Asan, Carayon, Beasley, \& Montague, 2015) that investigated factors that influence providers' screen sharing behaviors in primary care encounters; providers in this work did not want the patient to see the screen when they were looking at a psychiatrist's note or when they were documenting embarrassing information or legal issues.

\subsection{Workflow}

The WIS instrument, or similar workflow integration assessment tools, have not been used in previous studies of exam room layout. The three WIS subscales of navigation, usability, and workload, as well as overall WIS scores, indicated a significant difference between layouts, with Layout B having better scores. Moreover, providers rated Layout B higher, meaning that they believed layout B was easier to incorporate into their clinical workflow rather than layout A. The debrief interviews are helpful for interpreting these results. Providers mentioned that layout A involves having their back to the patient and thus made interacting with the EHR and the patient very difficult. In contrast, with layout B, focusing between the EHR and the patient was nearly seamless, involving a simple shift in eye gaze. This easy shift in attention allowed providers to make changes in the EHR and talk to the patient with ease without having to change positions, which may have led to layout B having a more favorable WIS score. The one 
395 subscale of the WIS that was not statistically different was 'paper-based workarounds', but trended

396 towards significance. The lack of difference for this subscale may be the result of the simulation

397 environment; provider did not have access to any paper materials aside from a one-page overview of the

398 patient scenario and a list of medications provided by the patient. Transposing this study to a real-world

399 scenario, it is possible that over time certain paper-based workarounds would be developed.

400 4.5. Situation Awareness

Our assessment of changes in providers' situation awareness with different exam room layouts is,

403 we believe, novel in the existing literature. There was a higher perceived level of situation awareness

404 with layout B. Situation awareness was most likely facilitated in layout B again because of the flexibility

405 of the wall mount. The mounting system allows for the provider to have the patient in their peripheral

406 vision. This gives the provider freedom to change eye gaze from the EHR and patient quickly, but also

407 enables the provider to visually sense a disturbance with the patient when focused on the EHR and vice

408 versa. With layout A, if a provider needs to visually check the patient, they would need to either move

409 their body to put the patient within eye gaze, or move the patient next to them.

\section{$410 \quad$ 4.6. Debrief Interviews}

411 Debrief interview results were organized into major themes of layout preference, provider-patient

412 interaction, and redesign recommendations. Providers preferred layout B because it facilitated (1)

413 conversation; (2) maintaining eye contact with the patient while typing; (3) talking to the patient while

414 gazing at the computer; and (4) using a postural style that allows the clinician to face the patient most of

415 the time. This is consistent with several practices for promoting provider-patient interaction with the use

416 of exam room computing outlined by Patel et al. (2017), including using the computer to mediate

417 conversation. Indeed, layout B here, which included the wall-mounted monitor for ease of (re)-

418 positioning, allowed for a "joint focus of attention" (Frankel \& Saleem, 2013) that seems to allow the

419 provider to better manage the medical encounter. Just as an aviation pilot relies on an external field of

420 view as well as the instrument panel during complex coordinated actions, the medical provider can 
421 achieve the same joint focus of attention with the patient and the EHR when the layout allows for

422 positioning of the computer monitor in close proximity with the patient.

423

424

425

426

427

428

429

430

431

432

433

434

435

436

437 unique compared to previous studies. Previous work has demonstrated that an exam room wherein the

438 provider can readily share the computer screen can facilitate direct interaction and communication with

439 the patient; however, these studies were mainly qualitative (e.g., Chen, Ngo, Harrison, \& Duong, 2011;

440 Frankel et al., 2005; Ventres, Kooienga, Marlin, Vuckovic, \& Stewart, 2005).

442 set-up (layout B) from an objective point of view, but reducing the physicians perceived workload and

443 increasing situation awareness with a more thoughtful computing arrangement can lead to an increase in

444 patient centeredness and perhaps even patient care. This can mainly be achieved through screen sharing

445 by inviting the patient in on care decisions as they relate to the information on the EHR screen and giving

446 the patient a feeling of greater involvement. 
This study has some limitations that should be noted. Due to the challenges of recruiting

448 physicians to participate in a laboratory simulation away from their clinics, convenience sampling was

449 used and the majority of the participants were resident physicians, whose practices may not generalize to

450 all primary care providers. Although some of the providers had previous experience using a wall-

451 mounted armature system, which may have introduced some learning bias, there was a good deal of

452 variety in overall previous experiences with exam room computing set-ups across the providers.

453 Limitations of the current study also existed with the patient scenarios. The scenarios did not require the

454 provider to conduct a full physical exam, which would be more common for providers when conducting a

455 patient visit. However, this was omitted because the focus of the study was on the computing arrangement

456 and patient centeredness, not the provider's ability to conduct a physical examination. Additionally,

457 certain nuances of the provider-patient interaction, such as mutual eye gaze of the provider and patient on

458 the computer monitor, were not considered as part of patient centeredness, but should be incorporated in

459 future studies. Another limitation was that one of the study team members played the role of the patient in

460 each patient visit, could possibly have introduced bias during the study sessions. This was done because

461 hiring an independent patient actor was cost prohibitive for the study. However, the study team member

462 who played the patient was the senior member of the study team and took great care to be consistent

463 across layout types and providers, and not compel the provider to share the screen with them by following

464 a pre-determined patient file and pre-planned responses. Also, in both patient scenarios the patient was

465 interested in viewing trends of their blood pressure or respiratory rate values over a period of time. This

466 was purposefully designed into the scenarios to encourage the provider to share the screen at least once

467 while using layouts A and B. In reality, there are patients who may not be interested in viewing the

468 screen at all, which potentially limits the generalizability of the current laboratory simulation.

469 Finally, it would be interesting to see how layout A and B compare performance-wise over the

470 course of an entire work day. Future research should look to conduct studies of provider-patient scenarios

471 over the course of an entire work day in a real-world clinical environment. More specifically, future work

472 should focus on the effects of the different layouts on performance, patient centeredness, workload, 
473 workflow integration, and situation awareness over the course of multiple patient interactions, to

474 determine more realistic outcomes of the different layouts. Additionally, future studies could introduce a

475 patient scenario where providers are required to reference imaging data (X-rays, CT scans, etc.) to better

476 understand the role of the computing device in a more complex patient visit. Based on the study findings,

477 we argue that layout B would be preferred based on the lower amount of perceived workload, greater

478 perceived levels of situation awareness, and greater workflow integration. This may lead to providers

479 feeling less fatigued towards the end of the day. The conclusion about layout B as preferred, however, is

480 based solely on the study findings and does not take into account cost or other organizational factors.

\section{CONCLUSION}

485 (efficiency, errors, and proportion of time focused on the patient), results show that layout B was the 486 preferred exam room computing layout. Additionally, providers experienced reduced workload,

487 increased situation awareness, and better integration with clinical workflow using layout B when 488 compared to layout A. Layout B also encourages a greater amount of screen sharing activities, consistent 489 with the evolving paradigm of the computer and EHR being a third party and serving as a mediator 490 between provider and patient. This study partially supports our hypothesized expectations, but further

491 research is needed that focuses on the effects of each layout throughout multiple provider-patient 492 interactions over the course of an entire workday. We will conduct such a study with the same layouts 493 that exist in a live clinic setting as part of this funded work, documenting real patients' perspectives and 494 preferences, in addition to collecting provider data.

\section{REFERENCES} physicians' screen sharing behaviors in primary care encounters. International Journal of Medical Informatics, 84(10), 791-798. 
Asan, O., D Smith, P., \& Montague, E. (2014). More screen time, less face time-implications for EHR design. Journal of Evaluation in Clinical Practice, 20(6), 896-901.

Baker, L. H., Reifsteck, S. W., \& Mann, W. R. (2003). Connected: communication skills for nurses using the electronic helath record. Nurs. Econ., 21, 85-88.

Chen, Y., Ngo, V., Harrison, S., \& Duong, V. (2011). Unpacking exam-room computing: negotiating computer-use in patient-physician interactions. In Proceedings of the SIGCHI Conference on Human Factors in Computing Systems (pp. 3343-3352). ACM.

Endsley, M. R. (1995). Toward a Theory of Situation Awareness in Dynamic Systems. Human Factors, $37,32-64$.

Flanagan, M., Arbuckle, N., Saleem, J. J., Militello, L. G., Haggstrom, D. A., \& Doebbeling, B. N. (2011). Development of a workflow integration survey (WIS) for implementing computerized clinical decision support. AMIA Annual Symposium Proceedings, 427-434.

Frankel, R. M. (2016). Computers in the examination room. JAMA Internal Medicine, 176(1), 128-129.

Frankel, R., Altschuler, A., George, S., Kinsman, J., Jimison, H., Robertson, N. R., \& Hsu, J. (2005). Effects of exam-room computing on clinician-patient communication. Journal of General Internal Medicine, 20(8), 677-682.

Frankel, R. M., \& Saleem, J. J. (2013). "Attention on the flight deck": What ambulatory care providers can learn from pilots about complex coordinated actions. Patient Education and Counseling, 93(3), 367-372.

Hart, S., \& Staveland, L. (1988). Development of the NASA-TLX (Task Load Index): Results of empirical and theoretical research. North-Holland: Elsevier Science Publishers.

Helfrich, C. D., Sylling, P. W., Gale, R. C., Mohr, D. C., Stockdale, S. E., Joos, S., . . Nelson, K. M. (2016). The facilitators and barriers associated with implementation of a patient-centered medical home in VHA. Implementation Science, 11, 24.

Kumarapeli, P., \& de, L. S. (2013). Using the computer in the clinical consultation; setting the stage, reviewing, recording, and taking actions: multi-channel video study. Journal of American Medical Informatics Association, 20, 67-75.

McGrath, J. M., Arar, N. H., \& Pugh, J. A. (2007). The influence of electronic medical record usage on nonverbal communication in the medical interview. Health Informatics Journal, 13, 105-118.

Montague, E., \& Asan, O. (2014). Dynamic modeling of patient and physician eye gaze to understand the effects of electronic health records on doctor-patient communication and attention. International Journal of Medical Informatics, 83, 225-234.

Montague, E., Xu, J., Chen, P. Y., Asan, O., Barrett, B. P., \& Chewning, B. (2011). Modeling eye gaze patterns in clinician-patient interaction with lag sequential analysis. Human Factors, 53, 502-516.

Patel, M. R., Vichich, J., Lang, I., Lin, J., \& Zheng, K. (2017). Developing an evidence base of best practices for integrating computerized systems into the exam room: a systematic review. Journal of American Medical Informatics Association, 24(1), 207-215.

Pearce, C., Arnold, M., Phillips, C., Trumble, S., \& Dwan, K. (2011). The patient and the computer in the primary care consultation. Journal of American Medical Informatics Association, 18, 138-142.

Pearce, C., Dwan, K., Arnold, M., Phillips, C., \& Trumble, S. (2009). Doctor, patient and computer--a framework for the new consultation. International Journal of Medical Informatics, 78, 32-38.

Rouf, E., Whittle, J., Lu, N., \& Schwartz, M. D. (2007). Computers in the exam room: differences in physician-patient interaction may be due to physician experience. Journal of General Internal Medicine, 22, 43-48.

Saleem, J. J., Flanagan, M. E., Russ, A. L., McMullen, C. K., Elli, L., Russell, S. A., , . Frankel, R. M. (2014). You and me and the computer makes three: variations in exam room use of the electronic health record. Journal of American Medical Informatics Association, 21, 147-151.

Sanders, M. S., \& McCormick, E. J. (1993). Human Factors in Engineering and Design. New York, NY: McGraw-Hill, Inc.

Selcon, S. J., \& Taylor, R. M. (1990). Evaluation of the situation awareness rating technique (SART) as a tool for aircrew systems design technique (SART) as a tool for aircrew systems design. Paper 
presented at the AGARD AMP symposium 'Situational Awareness in Aerospace Operations',

554 Ventres, W., Kooienga, S., Marlin, R., Vuckovic, N., \& Stewart, V. (2005). Clinician style and examination room computers: a video ethnography. Family Medicine, 37(4), 276-81.

Ventres, W., Kooienga, S., Vuckovic, N., Marlin, R., Nygren, P., \& Stewart, V. (2006). Physicians, patients, and the electronic health record: an ethnographic analysis. The Annals of Family Medicine, 6, 124-131. 